\title{
Determination the Role of Weeds Hosts in Spreading of Sheath Blight from Weeds to Rice Crop in Western Plain Zone of Uttar Pradesh, India
}

\author{
Mohd Ali ${ }^{1}$, Ramji Singh ${ }^{1}$, Mehi Lal ${ }^{2}$, Sorabh Chaudhary ${ }^{2}$, \\ Santosh Kumar ${ }^{3}$ and Shameem Ahmad ${ }^{1}$ \\ ${ }^{I}$ Department of Plant Pathology, SVP University of Agri. and Tech., Modipuram, \\ Meerut -250 110 (UP), India \\ ${ }^{2}$ Plant Protection Section, ICAR-Central Potato Research Institute Regional Station, \\ Modipuram, Meerut-250 110 (UP), India \\ ${ }^{3}$ Department of Plant Pathology, Bihar Agriculture University, Sabour, \\ Bhagalpur - 813210 (Bihar), India \\ *Corresponding author
}

\begin{tabular}{|c|}
\hline Keywords \\
\hline $\begin{array}{l}\text { Rice, Rhizoctonia } \\
\text { solani, Sheath } \\
\text { blight, Weeds, } \\
\text { Host range }\end{array}$ \\
\hline Article Info \\
\hline $\begin{array}{l}\text { Accepted: } \\
\text { 15 August } 2019 \\
\text { Available Online: } \\
\text { 10 September } 2019\end{array}$ \\
\hline
\end{tabular}

A B S T R A C T

Rice is a staple food for human being all over the world and India is the second largest producer and consumer of rice at global level. Production of rice in India is limited by several biotic and abiotic factors. Rice sheath blight (Rhizoctonia solani AG1-IA) is one of the most important factor causes significant grain yield and quality losses at worldwide. Yield losses of up to 50\% have been reported under most conducive environments in India. Several uncultivated (weeds) and cultivated plant species are known to act as alternate and collateral hosts of $R$. solani in different agro-climatic regions of India. The cv. Pusa Basmati-1 (PB-1) grown in the western plain region of Uttar Pradesh and to screen weed species growing in and around paddy fields to study the carryover of pathogen from rice to weeds and vice-versa. Out of 75 weed species tested for host range of $R$. solani, 73 were found to show high or low degree of symptoms in vitro. Whereas, 11 weed species i.e. Launaea procumbens, Cynodon dactylon, Echinochloa colona, Blumea lacera, Eragrostis tenella, Typha angustata, Chloris barbata, Xanthium strumarium, Cyperus rotundus, Paspalum distichum and Bidens pilosa were found to carry infection of $R$. solani as they exhibited characteristic symptoms after artificial inoculation. A total of 22 weed species were evaluated inside rice field using cv. PB-1 to know infection level and how associated in spreading the disease. Out of 22 weed species, 13 weed species viz. Echinochloa colona, Brachiaria decumbens, Cyperus rotundus, Setaria verticillata, Paspalum distichum, Leptochloa chinensis, Brachiaria reptans, Imperata cylindrica, Saccharum spontaneum, Digitaria sangunalis, Cyperus difformis, Eclipta alba and Cynodon dactylon showed higher disease incidence and five weed species i.e. Sorghum halepense, Echinochloa colona, Paspalum distichum, Imperata cylindrica and Desmostachya bipinnata were showed higher disease severity as well as a very prominent role in spreading $R$. solani to rice plants during both the years (2011 and 2012). Weed species Echinochloa colana and Brachiaria decumbens associated with rice were maximum percent disease severity $(15.62 \% \& 12.33 \%)$ and disease incidence $(25.47 \% \& 24.41 \%)$, respectively when planted in between the rows of rice under field condition. These weeds are having played a crucial role in transfer and spread of pathogen from weeds to rice crop. These studies will help in managing the sheath blight disease. 


\section{Introduction}

Rice (Oryza sativa L.) is a major food constituent of human diet, for more than two third population of India. Globally, after China, India ranks second in rice production. It is a major food crop in India, China and most of the other Asian countries, where 92\% of the world's rice is cultivated (Rai, 2006). To fulfill the rice requirement globally, it is estimated that about 115 million tons of additional milled rice needs to be produced by 2035, which is equivalent to an overall increase of $26 \%$ in the next 25 years. At present, rice productivity has been increased four times from the past five decades that made possible by the adoption of semi-dwarf high-yielding varieties (HYV) with applying modern cultural practices.

Efforts for enhancing the productivity are limited by a number of biotic and abiotic stresses. The rice crop suffers from a number of devastating diseases caused by fungi, bacteria, viruses, nematodes, phytoplasma. Alone fungal diseases are responsible for about 12 to $20 \%$ yield losses per year (Rajan, 1987). Among the fungal diseases, sheath blight caused by Rhizoctoina solani Kühn [Thanetephorus cucumeris (Frank) Donk] is one of the most serious threat for Basmati rice production in India, specially where acreage under Basmati rice is growing exponentially. $R$. solani is a complex of genetically distinct groups of fungi with more than 100 species; having very diverse life histories (Anderson 1982; Adams, 1988; Binder et al., 2005) with a wide host range and infects large number of crop plants, horticulture and weed species (Ou, 1972). Among R. solani anastomosis groups (AGs), the subgroup AG1-IA is one of the predominant causal agent of sheath blight from rice growing regions worldwide (Taheri et al., 2007; Bernardes-De-Assis et al., 2009; Gonzalez-Vera et al., 2010).
The impact of weed competition on rice yield has been well documented (Antralina et al., 2015; Sureshkumar et al., 2016; Hosoya and Sugiyama, 2017). Weeds have also been identified as hosts for a variety of rice pathogens including Ustilaginoidea virens (Shetty and Shetty, 1987; Deng et al., 2015), Fusarium fujikuroi (Carter et al., 2008), Xanthomonas oryzae pv. oryzae (Brar and Thind, 1994), and Bipolaris oryzae (Biswas and Mohanty, 1995). As alternative hosts for pytopathogens, weeds contribute to increased inoculums density, allow carryover of pathogens in crop rotation, and provide a base for pathogenic variation (Hartman et al., 1986). Several uncultivated (weeds) and cultivated plant species are also known to act as alternate and collateral hosts of $R$. solani in different agro-climatic regions of India (Singh et al., 2012). Various weeds like Cyperus rotundus, Cyperus difformis, Cynodon dactylon, Echinochloa colonum, Commelina obliqua and Amaranthus viridis were reported as collateral hosts and the pathogen survives in these hosts in absence of rice plants (Acharya and Sengupta, 1998).

The host range studies of $R$. solani indicated that it was successfully infected 14 different plant species belonging to three families. This indicates that weeds and plants species serve as collateral hosts and responsible for spreading the disease in next crop season (Srinivas et al., 2014). As a consequence, the increasing importance of the disease and lack of information on weed hosts of $R$. solani in the Uttar Pradesh, there is a need to gather information on the off season survival of $R$. solani in rice ecosystem on different weeds and plant species. The present investigation is carried out to record host range of $R$. solani on different weeds, the infection level of sheath blight in different weeds grown in and around paddy fields to study their carryover of pathogen from rice to weeds and vice-versa in the Uttar Pradesh. 


\section{Materials and Methods}

\section{Evaluation of host range of $R$. solani on different weed species}

Seventy five weed species belonging to different families were selected for the host range study. The leaves of different weed species were collected in polythene bags, labeled and stored. Leaves were cut with sterilized scissor $(90.0 \%$ alcohol) and placed on sterilized and moist Whatman filter paper Grade 1 in the sterilized petriplates. Mycelial bits of $5 \mathrm{~mm}$ dia. were cut from the actively growing colony of $R$. solani and placed on the detached weed leaves in the petriplates containing moist and sterilized Whatman filter paper (Kumar et al., 2013).

Three replications were maintained for each treatment. Inoculated leaves were incubated in BOD at $28+2^{\circ} \mathrm{C}$. Three leaves were inoculated in each petriplates. After inoculation, infection or no infection were observed at 24 hours intervals for one week. Development of lesions on the detached leaves was considered to be the host for $R$. solani.

\section{Determination of role of different weed species in spread of sheath blight in rice}

To determine the role of various weed species in spread of sheath blight disease, a field experiment was conducted at Chirori Farm, S. V Patel Agriculture University, Meerut UP, for two crop season i.e. Kharif 2011 to Kharif 2012.

A total of 22 weed species were evaluated inside rice field. Weed species were planted in between the two rows of rice and inoculated with $R$. solani inoculum grown on rice bran. Observations on sheath blight disease incidence and severity were recorded after every 25 days following Standard Evaluation System for Rice (IRRI, 1996).

\section{Results and Discussion}

Determination of host range of $R$. solani on different weed species in vitro

Host range studies revealed that $R$. solani successfully colonized all the tested weed species except Datura metel and Commelina benghalensis and produced typical symptoms of sheath blight under artificial in vitro inoculation (Table 1). The sheath blight symptoms were started appearing 7 days post inoculation. The weed species Launaea procumbens, Cynodon dactylon, Echinochloa colona, and Blumea lacera recorded highest disease severity (range $81.43 \%$ to $86.95 \%$ ), followed by Eragrostis tenella, Typha angustata, Chloris barbata, Xanthium strumarium, Cyperus rotundus, Paspalum distichum and Bidens pilosa (range 70.48\%$77.90 \%$ ) and produced typical symptoms of sheath blight. However, six weed species viz., Tridax procumbens, Eleusine indica, Leptochloa chinensis, Setaria verticillata, Ipomoea cairica and Dactyloctenium aegyptium showed moderately high disease severity (range $60.24 \%-67.33 \%$ ) and 26 weed species namely Alternanthera sessilis, Rhynchosia minima, Desmostachya bipinnata, Cassia tora, Lantana camara, Euphorbia hirta, Vitis carnosa, Peristrophe paniculata, Croton bonplandianum, Alternanthera pungens, Echinochloa glabrescens, Eclipta alba, Coccinia grandis, Anisomeles indica, Ocimum basilicum, Setaria verticillata, Brachiaria reptans, Triumfetta rhomboide, Malvastrum coromandelianum, Mecardonia procumbens, Polygonum hydropiper, Erianthus munja, Leucas cephalotes, Sorghum halepense, Sida acuta, and Kirganelia reticulate showed moderately low disease severity (range 30.57\%-57.62\%). The remaining 30 weed species Solanum nigrum, Celosia argentea, Scoparia dulcis, Trianthema monogyna, Withania somnifera, Brachiaria decumbens, Tribulus terrestris, Parthenium 
hysterophorus, Mukia maderaspatana, Cyperus difformis, Oxalis corniculata, Cannabis sativa, Cissampelos hirsute, Ziziphus mauritiana, Anisomeles malabarica, Lindernia crustacean, Calotropis procera, Ageratum houstonianum, Digera muricata, Chenopodium album, Alysicarpus vaginalis, Saccharum spontaneum, Phyllanthus niruri, Physalis minima, Urena lobata, Cuscuta reflexa, Sida rhombifolia, Achyranthes aspera, Cassia occidentalis and Amaranthus viridis showed lower disease severity (range 0.95\%$28.38 \%$ ). The pathogen was re-isolated from all the infected weed species which produced typical characteristics of $R$. solani on potato dextrose agar (PDA). The results indicate that weed species serve as subsidiary hosts (alternate/collateral host) of $R$. solani and helped in the disease incidence in the next cropping season. Similar observations were reported by Tsai (1974) and found that 20 species of weeds from 11 families infected with $R$. solani and that the sclerotia obtained from the diseased tissues of weeds produce typical symptoms of sheath blight of rice. The occurrence of fungus $R$. solani on several weeds in nature was also observed by several other workers (Roy, 1973; Sharma and Mukherjee, 1978). Srinivas et al. (2014) reported that rice isolates of $R$. solani infected 14 species belonging to three different families i.e. Amaranthaceae, Graminaceae and Leguminaceae.

\section{Determination of role of different weed species in spread of sheath blight in rice}

\section{Effect on percent disease severity}

To find out the role of different weed species in spreading the sheath blight of rice. An experiment was conducted under field condition using 22 different weed species. Out of 22 weed species, six weed species i.e. Echinochloa colona, Brachiaria decumbens, Imperata cylindrica, Leptochloa chinensis,
Brachiaria reptans, and Eleusine indica were found to have a very prominent role in spreading the pathogen i.e. R. solani to rice plants, as there was considerable level of disease severity (range 10.23\%-15.62\%) of sheath blight in rice during both the years (2011 and 2012) planted in association with these five weeds (Table 2).

When weed species Echinochloa colona was grown in between two rows of PB-1, it was observed that this resulted in highest disease severity of $20.83 \%$ on PB-1. The rice associated with weed Echinochloa colona also showed higher infection of sheath blight of rice, except Leptochloa chinensis which was free from the disease but rice row associated with this weed also showed some minimum level of disease severity (13.89\%) as compared to above mentioned other weeds. During 2012 the disease severity was comparatively lower than previous years as, out of 22 weed species, 8 weed species viz., Echinochloa colona (10.42\%) and Paspalum distichum (7.64\%) showed comparatively lower severity of sheath blight among these weeds. The higher average severity of sheath blight of rice on variety PB-1 in association with weed species Echinochloa colona, Brachiaria decumbens, Brachiaria reptans, Paspalum distichum, Saccharum spontaneum, Imperata cylindrica, Eleusine indica, Cyperus difformis and Cynodon dactylon, clearly indicate that these weeds are having greater role in spreading sheath blight disease to rice plants. Kannaiyan and Prasad (1980) have listed 30 monocot weed species as host of Thanatephorus cucumeries (Rhizoctnia solani). Kozaka (1965) and Tsai (1974) observed that rice fungus infected 20 species which are from 11 families and observed that the sclerotia from diseased tissue of weed hosts produced typical symptoms of sheath blight on paddy. Kozaka (1961) from Japan recorded 188 species of plants from 32 families that can be infected by this fungus. 
Two virulent isolates of Thanatephorus cucumeris could infect and survive on several weed hosts which are commonly found in rice fields namely Echinocloa crusgalli, $E$. colonum, Fimbristylis littoralis, Cyperus rotandus. Kannaiyan and Prasad (1980) have listed 30 monocot weed species as host of Thanatephorus cucumeris.

Table.1 Evaluation of host range of $R$. solani on different weed species in-vitro in Uttar Pradesh

\begin{tabular}{|l|l|l|c|c|}
\hline $\begin{array}{c}\text { S. } \\
\text { No. }\end{array}$ & \multicolumn{1}{|c|}{ Botanical name } & \multicolumn{1}{|c|}{ Family } & $\begin{array}{c}\text { Total weed leaves } \\
\text { height }\end{array}$ & $\begin{array}{c}\text { Per-cent disease } \\
\text { severity }\end{array}$ \\
\hline $\mathbf{1}$ & Datura metel & Solanaceae & 5 & 0.00 \\
\hline $\mathbf{2}$ & Commelina benghalensis & Commelinaceae & 5 & 0.00 \\
\hline $\mathbf{3}$ & Solanum nigrum & Solanaceae & 5 & 0.95 \\
\hline $\mathbf{4}$ & Celosia argentea & Amaranthaceae & 5 & 1.76 \\
\hline $\mathbf{5}$ & Scoparia dulcis & Scrophulariaceae & 5 & 3.38 \\
\hline $\mathbf{6}$ & Trianthema monogyna & Aizoaceae & 5 & 5.71 \\
\hline $\mathbf{7}$ & Withania somnifera & Solanaceae & 5 & 5.71 \\
\hline $\mathbf{8}$ & Brachiaria decumbens & Poaceae & 5 & 6.00 \\
\hline $\mathbf{9}$ & Tribulus terrestris & Zygophyllaceae & 5 & 6.86 \\
\hline $\mathbf{1 0}$ & Parthenium hysterophorus & Asteraceae & 5 & 7.43 \\
\hline $\mathbf{1 1}$ & Mukia maderaspatana & Cucurbitaceae & 5 & 7.57 \\
\hline $\mathbf{1 2}$ & Cyperus difformis & Cyperaceae & 5 & 7.62 \\
\hline $\mathbf{1 3}$ & Oxalis corniculata & Oxalidaceae & 5 & 7.67 \\
\hline $\mathbf{1 4}$ & Cannabis sativa & Cannabaceae & 5 & 10.56 \\
\hline $\mathbf{1 5}$ & Cissampelos hirsute & Menispermaceae & 5 & 10.57 \\
\hline $\mathbf{1 6}$ & Ziziphus mauritiana & Rhamnaceae & 5 & 11.24 \\
\hline $\mathbf{1 7}$ & Anisomeles malabarica & Lamiaceae & 5 & 11.90 \\
\hline $\mathbf{1 8}$ & Lindernia crustacean & Scrophulariaceae & 5 & 15.90 \\
\hline $\mathbf{1 9}$ & Calotropis procera & Asclepiadaceae & 5 & 16.43 \\
\hline $\mathbf{2 0}$ & Ageratum houstonianum & Asteraceae & 5 & 17.14 \\
\hline $\mathbf{2 1}$ & Digera muricata & Amaranthaceae & 5 & 17.33 \\
\hline $\mathbf{2 2}$ & Chenopodium album & Chenopodiaceae & 5 & 17.90 \\
\hline $\mathbf{2 3}$ & Alysicarpus vaginalis & Fabaceae & 5 & 20.24 \\
\hline $\mathbf{2 4}$ & Saccharum spontaneum & Poaceae & 5 & 23.52 \\
\hline $\mathbf{2 5}$ & Phyllanthus niruri & Euphorbiaceae & 5 & 23.52 \\
\hline $\mathbf{2 6}$ & Physalis minima & Solanaceae & 5 & 25.38 \\
\hline $\mathbf{2 7}$ & Urena lobata & Malvaceae & 5 & 25.62 \\
\hline $\mathbf{2 8}$ & Cuscuta reflexa & Cuscutaceae & 5 & 26.05 \\
\hline $\mathbf{2 9}$ & Sida rhombifolia & Malvaceae & 5 & 28.95 \\
\hline $\mathbf{3 0}$ & Achyranthes aspera & Amaranthaceae & 5 & 30.57 \\
\hline $\mathbf{3 1}$ & Cassia occidentalis & Fabaceae & 5 & 33.43 \\
\hline $\mathbf{3 2}$ & Amaranthus viridis & Amaranthaceae & 5 & \\
\hline $\mathbf{3 3}$ & Alternanthera sessilis & Amaranthaceae & 5 & \\
\hline $\mathbf{3 4}$ & Rhynchosia minima & Fabaceae & 5 & \\
\hline & & & & \\
\hline
\end{tabular}


Int.J.Curr.Microbiol.App.Sci (2019) 8(9): $972-982$

\begin{tabular}{|c|c|c|c|c|}
\hline 35 & Desmostachya bipinnata & Poaceae & 5 & 33.48 \\
\hline 36 & Cassia tora & Fabaceae & 5 & 33.86 \\
\hline 37 & Lantana camara & Verbenaceae & 5 & 34.05 \\
\hline 38 & Euphorbia hirta & Euphorbiaceae & 5 & 35.10 \\
\hline 39 & Vitis carnosa & Vitaceae & 5 & 36.29 \\
\hline 40 & Peristrophe paniculata & Acanthaceae & 5 & 39.10 \\
\hline 41 & Croton bonplandianum & Euphorbiaceae & 5 & 39.24 \\
\hline 42 & Alternanthera pungens & Amaranthaceae & 5 & 42.71 \\
\hline 43 & Echinochloa glabrescens & Poaceae & 5 & 43.00 \\
\hline 44 & Eclipta alba & Asteraceae & 5 & 43.00 \\
\hline 45 & Coccinia grandis & Cucurbitaceae & 5 & 44.29 \\
\hline 46 & Anisomeles indica & Lamiaceae & 5 & 44.76 \\
\hline 47 & Ocimum basilicum & Lamiaceae & 5 & 44.81 \\
\hline 48 & Setaria verticillata & Poaceae & 5 & 45.24 \\
\hline 49 & Brachiaria reptans & Poaceae & 5 & 45.57 \\
\hline 50 & Triumfetta rhomboidea & Tiliaceae & 5 & 47.29 \\
\hline 51 & Malvastrum coromandelianum & Malvaceae & 5 & 49.05 \\
\hline 52 & Mecardonia procumbens & Scrophulariaceae & 5 & 50.00 \\
\hline 53 & Polygonum hydropiper & Polygonaceae & 5 & 52.38 \\
\hline 54 & Erianthus munja & Poaceae & 5 & 52.76 \\
\hline 55 & Leucas cephalotes & Lamiaceae & 5 & 55.33 \\
\hline 56 & Sorghum halepense & Poaceae & 5 & 55.48 \\
\hline 57 & Sida acuta & Malvaceae & 5 & 56.10 \\
\hline 58 & Kirganelia reticulate & Euphorbiaceae & 5 & 57.62 \\
\hline 59 & Tridax procumbens & Asteraceae & 5 & 60.24 \\
\hline 60 & Eleusine indica & Poaceae & 5 & 61.43 \\
\hline 61 & Leptochloa chinensis & Poaceae & 5 & 62.29 \\
\hline 62 & Setaria verticillata & Poaceae & 5 & 63.05 \\
\hline 63 & Ipomoea cairica & Convolvulaceae & 5 & 64.52 \\
\hline 64 & Dactyloctenium aegyptium & Poaceae & 5 & 67.33 \\
\hline 65 & Eragrostis tenella & Poaceae & 5 & 70.48 \\
\hline 66 & Typha angustata & Typhaceae & 5 & 70.86 \\
\hline 67 & Chloris barbata & Poaceae & 5 & 71.14 \\
\hline 68 & Xanthium strumarium & Asteraceae & 5 & 75.14 \\
\hline 69 & Cyperus rotundus & Cyperaceae & 5 & 75.33 \\
\hline 70 & Paspalum distichum & Poaceae & 5 & 76.00 \\
\hline 71 & Bidens pilosa & Asteraceae & 5 & 77.90 \\
\hline 72 & Launaea procumbens & Asteraceae & 5 & 81.43 \\
\hline 73 & Cynodon dactylon & Poaceae & 5 & 81.57 \\
\hline 74 & Echinochloa colona & Poaceae & 5 & 84.02 \\
\hline \multirow[t]{2}{*}{75} & Blumea lacera & Asteraceae & 5 & 86.95 \\
\hline & $\mathrm{CD}(\mathrm{p}=0.05)$ & & & 9.772 \\
\hline
\end{tabular}


Table.2 Assessment of role of different weed species in spreading of sheath blight in rice cv. PB -1

\begin{tabular}{|c|c|c|c|c|c|c|c|c|}
\hline \multirow[t]{2}{*}{ S. No. } & \multirow[t]{2}{*}{ Weed botanical name } & \multirow[t]{2}{*}{ Weed family } & \multicolumn{3}{|c|}{$\begin{array}{c}\text { Percentage of disease severity on } \\
\text { rice }\end{array}$} & \multicolumn{3}{|c|}{$\begin{array}{l}\text { Percentage of disease } \\
\text { incidence on rice }\end{array}$} \\
\hline & & & 2011 & 2012 & Average & 2011 & 2012 & Average \\
\hline 1 & Sorghum halepense & Poaceae & $\begin{array}{c}3.12 \\
(9.30)^{*}\end{array}$ & $\begin{array}{c}1.74 \\
(7.34)\end{array}$ & $\begin{array}{c}2.43 \\
(8.32)\end{array}$ & $\begin{array}{c}10.40 \\
(16.60)\end{array}$ & $\begin{array}{c}7.90 \\
(15.14)\end{array}$ & $\begin{array}{c}9.15 \\
(15.87)\end{array}$ \\
\hline 2 & Commelina benghalensis & Commelinaceae & $\begin{array}{c}3.82 \\
(10.55)\end{array}$ & $\begin{array}{c}0.35 \\
(4.73)\end{array}$ & $\begin{array}{l}2.08 \\
(7.64)\end{array}$ & $\begin{array}{c}12.40 \\
(18.40)\end{array}$ & $\begin{array}{c}3.98 \\
(10.17)\end{array}$ & $\begin{array}{c}8.19 \\
(14.29)\end{array}$ \\
\hline 3 & Dactyloctenium aegyptium & Poaceae & $\begin{array}{c}10.27 \\
(17.12)\end{array}$ & $\begin{array}{c}0.35 \\
(4.73)\end{array}$ & $\begin{array}{c}5.21 \\
(10.93)\end{array}$ & $\begin{array}{c}19.11 \\
(23.67)\end{array}$ & $\begin{array}{c}2.61 \\
(7.75)\end{array}$ & $\begin{array}{c}10.86 \\
(15.71)\end{array}$ \\
\hline 4 & Brachiaria reptans & Poaceae & $\begin{array}{c}17.36 \\
(22.53)\end{array}$ & $\begin{array}{c}3.13 \\
(9.47)\end{array}$ & $\begin{array}{c}10.24 \\
(16.00)\end{array}$ & $\begin{array}{c}23.62 \\
(26.60)\end{array}$ & $\begin{array}{c}7.23 \\
(14.50)\end{array}$ & $\begin{array}{c}15.42 \\
(20.55)\end{array}$ \\
\hline 5 & Echinochloa colona & Poaceae & $\begin{array}{c}20.83 \\
(26.51)\end{array}$ & $\begin{array}{c}10.42 \\
(18.26)\end{array}$ & $\begin{array}{c}15.62 \\
(22.38)\end{array}$ & $\begin{array}{c}32.59 \\
(34.44)\end{array}$ & $\begin{array}{c}18.36 \\
(25.17)\end{array}$ & $\begin{array}{c}25.47 \\
(29.81)\end{array}$ \\
\hline 6 & Brachiaria decumbens & Poaceae & $\begin{array}{c}14.58 \\
(22.10)\end{array}$ & $\begin{array}{c}10.07 \\
(18.13)\end{array}$ & $\begin{array}{c}12.33 \\
(20.12)\end{array}$ & $\begin{array}{c}31.07 \\
(33.49)\end{array}$ & $\begin{array}{c}17.75 \\
(24.71)\end{array}$ & $\begin{array}{c}24.41 \\
(29.10)\end{array}$ \\
\hline 7 & Cynodon dactylon & Poaceae & $\begin{array}{c}5.55 \\
(12.81)\end{array}$ & $\begin{array}{c}7.29 \\
(14.46)\end{array}$ & $\begin{array}{c}6.42 \\
(13.64)\end{array}$ & $\begin{array}{c}20.41 \\
(24.34)\end{array}$ & $\begin{array}{c}10.25 \\
(17.25)\end{array}$ & $\begin{array}{c}15.33 \\
(20.80)\end{array}$ \\
\hline 8 & Setaria verticillata & Poaceae & $\begin{array}{c}7.27 \\
(14.60)\end{array}$ & $\begin{array}{c}8.68 \\
(15.89)\end{array}$ & $\begin{array}{c}7.98 \\
(15.25)\end{array}$ & $\begin{array}{c}25.30 \\
(29.83)\end{array}$ & $\begin{array}{c}15.07 \\
(22.58)\end{array}$ & $\begin{array}{c}20.18 \\
(26.21)\end{array}$ \\
\hline 9 & Paspalum distichum & Poaceae & $\begin{array}{c}10.06 \\
(18.24)\end{array}$ & $\begin{array}{c}7.64 \\
(16.00)\end{array}$ & $\begin{array}{c}8.85 \\
(17.12)\end{array}$ & $\begin{array}{c}24.69 \\
(29.37)\end{array}$ & $\begin{array}{c}15.60 \\
(23.01)\end{array}$ & $\begin{array}{c}20.14 \\
(26.19)\end{array}$ \\
\hline 10 & Saccharum spontaneum & Poaceae & $\begin{array}{c}9.03 \\
(16.60)\end{array}$ & $\begin{array}{c}7.98 \\
(16.29)\end{array}$ & $\begin{array}{c}8.51 \\
(16.45)\end{array}$ & $\begin{array}{c}21.44 \\
(25.03)\end{array}$ & $\begin{array}{c}18.51 \\
(25.29)\end{array}$ & $\begin{array}{c}19.98 \\
(25.16)\end{array}$ \\
\hline 11 & Cyperus rotundus & Cyperaceae & $\begin{array}{c}8.33 \\
(16.69)\end{array}$ & $\begin{array}{c}6.59 \\
(13.89)\end{array}$ & $\begin{array}{c}7.46 \\
(15.29)\end{array}$ & $\begin{array}{c}26.81 \\
(30.76)\end{array}$ & $\begin{array}{c}21.70 \\
(26.89)\end{array}$ & $\begin{array}{c}24.26 \\
(28.82)\end{array}$ \\
\hline 12 & Digitaria sangunalis & Poaceae & $\begin{array}{c}10.42 \\
(18.14)\end{array}$ & $\begin{array}{c}5.54 \\
(12.77)\end{array}$ & $\begin{array}{c}7.98 \\
(15.45)\end{array}$ & $\begin{array}{c}21.08 \\
(27.06)\end{array}$ & $\begin{array}{c}18.18 \\
(23.10)\end{array}$ & $\begin{array}{c}19.63 \\
(25.08)\end{array}$ \\
\hline 13 & Eleusine indica & Poaceae & $\begin{array}{c}13.54 \\
(21.33)\end{array}$ & $\begin{array}{c}6.93 \\
(15.14)\end{array}$ & $\begin{array}{c}10.23 \\
(18.23)\end{array}$ & $\begin{array}{c}15.28 \\
(22.27)\end{array}$ & $\begin{array}{c}16.63 \\
(22.95)\end{array}$ & $\begin{array}{c}15.95 \\
(22.61)\end{array}$ \\
\hline 14 & Imperata cylindrica & Poaceae & $\begin{array}{c}15.63 \\
(23.25)\end{array}$ & $\begin{array}{c}8.68 \\
(16.86)\end{array}$ & $\begin{array}{c}12.15 \\
(20.05)\end{array}$ & $\begin{array}{c}22.87 \\
(28.53)\end{array}$ & $\begin{array}{c}17.30 \\
(24.28)\end{array}$ & $\begin{array}{c}20.08 \\
(26.40)\end{array}$ \\
\hline 15 & Leptochloa chinensis & Poaceae & 13.89 & 7.29 & 10.59 & 23.84 & 20.53 & 22.18 \\
\hline
\end{tabular}


Int.J.Curr.Microbiol.App.Sci (2019) 8(9): 972-982

\begin{tabular}{|c|c|c|c|c|c|c|c|c|}
\hline & & & $(21.79)$ & $(15.21)$ & $(18.50)$ & $(28.91)$ & $(25.42)$ & $(27.16)$ \\
\hline 16 & Phyllanthus niruri & Euphorbiaceae & $\begin{array}{c}6.60 \\
(14.58)\end{array}$ & $\begin{array}{c}11.81 \\
(20.06)\end{array}$ & $\begin{array}{c}9.20 \\
(17.32)\end{array}$ & $\begin{array}{c}14.61 \\
(21.71)\end{array}$ & $\begin{array}{c}20.52 \\
(26.13)\end{array}$ & $\begin{array}{c}17.57 \\
(23.92)\end{array}$ \\
\hline 17 & Cyperus difformis & Cyperaceae & $\begin{array}{c}5.21 \\
(12.37)\end{array}$ & $\begin{array}{c}11.11 \\
(19.25)\end{array}$ & $\begin{array}{c}8.16 \\
(15.81)\end{array}$ & $\begin{array}{c}14.04 \\
(21.74)\end{array}$ & $\begin{array}{c}11.63 \\
(19.62)\end{array}$ & $\begin{array}{c}12.83 \\
(20.68)\end{array}$ \\
\hline 18 & Eclipta alba & Asteraceae & $\begin{array}{c}6.60 \\
(13.04)\end{array}$ & $\begin{array}{c}5.90 \\
(12.70)\end{array}$ & $\begin{array}{c}6.25 \\
(12.87)\end{array}$ & $\begin{array}{c}12.97 \\
(19.01)\end{array}$ & $\begin{array}{c}6.04 \\
(11.92)\end{array}$ & $\begin{array}{c}9.51 \\
(15.46)\end{array}$ \\
\hline 19 & Launea nudicaulis & Asteraceae & $\begin{array}{c}4.51 \\
(11.56)\end{array}$ & $\begin{array}{c}7.97 \\
(14.67)\end{array}$ & $\begin{array}{c}6.24 \\
(13.12)\end{array}$ & $\begin{array}{c}4.67 \\
(10.81)\end{array}$ & $\begin{array}{c}13.80 \\
(19.49)\end{array}$ & $\begin{array}{c}9.24 \\
(15.15)\end{array}$ \\
\hline 20 & Desmostachya bipinnata & Poaceae & $\begin{array}{c}2.08 \\
(7.23)\end{array}$ & $\begin{array}{c}7.96 \\
(16.30)\end{array}$ & $\begin{array}{c}5.02 \\
(11.77)\end{array}$ & $\begin{array}{c}2.40 \\
(7.56)\end{array}$ & $\begin{array}{c}18.72 \\
(25.34)\end{array}$ & $\begin{array}{c}10.56 \\
(16.45)\end{array}$ \\
\hline 21 & Chloris sp. & Poaceae & $\begin{array}{c}0.00 \\
(4.05)\end{array}$ & $\begin{array}{c}8.32 \\
(16.53)\end{array}$ & $\begin{array}{c}4.16 \\
(10.29)\end{array}$ & $\begin{array}{c}2.40 \\
(7.56)\end{array}$ & $\begin{array}{c}13.09 \\
(21.00)\end{array}$ & $\begin{array}{c}7.74 \\
(14.28)\end{array}$ \\
\hline \multirow[t]{3}{*}{22} & Vernonia gigantean & Asteraceae & $\begin{array}{c}0.00 \\
(4.05)\end{array}$ & $\begin{array}{c}4.16 \\
(10.72)\end{array}$ & $\begin{array}{c}2.08 \\
(7.39)\end{array}$ & $\begin{array}{c}0.00 \\
(4.05)\end{array}$ & $\begin{array}{c}3.81 \\
(9.95)\end{array}$ & $\begin{array}{c}1.91 \\
(7.00)\end{array}$ \\
\hline & Mean & & $\begin{array}{c}8.57 \\
(15.38)\end{array}$ & $\begin{array}{c}6.81 \\
(14.06)\end{array}$ & & $\begin{array}{c}17.36 \\
(22.35)\end{array}$ & $\begin{array}{c}13.60 \\
(20.08)\end{array}$ & \\
\hline & $\mathrm{CD}(\mathrm{p}=0.05)$ & & 8.36 & 6.92 & & 13.42 & 11.24 & \\
\hline
\end{tabular}

* Data in the parentheses are angular transformed values 


\section{Effect on percent disease incidence}

Out of 22 weed species, tested 13 weed species viz. Echinochloa colona, Brachiaria decumbens, Cyperus rotundus, Leptochloa chinensis, Setaria verticillata, Paspalum distichum, , Imperata cylindrica, Saccharum spontaneum, Digitaria sangunalis, Phyllanthus niruri, Eleusine indica, Brachiaria reptans and Cynodon dactylon showed higher disease incidence (range $25.47 \%-15.33 \%$ ) in association with rice during both the years (2011 and 2012) (Table 2). Other weed species i.e. Sorghum halepense, Launea nudicaulis, Desmostachya bipinnata, Chloris sp. and Vernonia gigantean were found to harbor lower incidence of sheath blight disease under field condition. The rice variety $\mathrm{PB}-1$ showed highest disease incidence of $32.59 \%$ in the rows between Echinochloa colona and Chloris sp. and Desmostachya bipinnata showed very less disease incidence $(2.40 \%)$ of sheath blight during 2011.

The weed species Cyperus rotundus $(21.70 \%)$ showed highest disease incidence and Dactyloctenium aegyptium (2.61\%) showed very less disease incidence of sheath blight during 2012. Thus there was higher average incidence of sheath blight in association with weed species Echinochloa colona, Brachiaria decumbens, Cyperus rotundus, Paspalum distichum, Leptochloa chinensis, Brachiaria reptans, Imperata cylindrica, Saccharum spontaneum, Digitaria sangunalis and Cynodon dactylon. Singh et al. (2012) determined the status of sheath blight in various rice cultivars and hybrids at maximum tillering to dough stage. The disease was particularly devastating on PR 114, PAU 201, Pusa Basmati-1121, Pusa Basmati-1, PA 6444 (Arize 6444), PA 6129 (Arize 6129) and CSR 30. The disease appeared in severe form particularly in PR 114, CSR 30 and Pusa Basmati-1121 due to build up of inoculum during last few years and prevalence of highly favorable environmental conditions for the disease development. They also reported that out of 28 weed species tested to determine the weed hosts of $R$. solani, a varied degree of disease development was recorded on 24 weeds following inoculation of the rice isolate while 4 weeds were not infected. All the $R$. solani isolates of weed hosts were crosspathogenic on rice cultivar HKR 127. Euphorbia microphylla, E. hirta, Cassia obtusifolia, Dichanthium annulatum, Caesulia axillaris, Sida sp., Xanthium strumarium, Parthenium hysterophorus, Chenopodium album and Trianthema portulacastrum are being reported as hosts of $R$. solani. The highest disease incidences of $R$. solani on weed species i.e. Echinocloa crusgalli and $E$. colonum have also been advocated by Kozaka (1965).

The present study identified 73 weed species that are potential hosts for $R$. solani AG1-IA. The ability of weeds to serve as hosts for the pathogen in the absence of rice may explain why epidemics can occur in fields where rice has not been cultivated previously. Wu et al. (2015) suggested that rational use of $\mathrm{N}$ rate and increased row spacing reduced the severity of sheath blight by promoting air movement through rice canopy. Wu et al. (2013) concluded that differences in canopy structure resulting from planting density and $\mathrm{N}$ rates influences sheath blight epidemics. High infestation of weed species may affect sheath blight development and incidence by increasing total plant density and reducing air movement within the rice canopy as well as serving as bridge hosts for pathogen. The role of weeds as hosts for the pathogens involved in the sheath blight may play an important role in disease epidemiology by providing another source of spread through the canopy other than rice to rice contact. Therefore, it is recommended that weeding at time intervals 
during cropping season and rotation of nonhost crop helps in minimizing the disease incidence in the next cropping season. The present study highlight the importance of weed control, not only for reducing plant competition and increasing production, but also for the potential impact on sheath blight development.

\section{Acknowledgements}

The authors are grateful to the Head, Department of Plant Pathology, SVP University of Ag. \& Tech., Modipuram, Meerut for providing necessary facilities for conducting the research and needful guidance.

\section{References}

Acharya, S. and Sengupta, P. K. 1998. Collateral hosts of rice sheath blight fungus Rhizoctonia solani. Oryza, 35: 89-90.

Adam, G. C., 1988. Thanatephorus cucumeris (Rhizoctonia solani) a species complex of wide host ranges. In G. S. Sidhu (Eds.), Advances in Plant Pathology, Academic Press, 535-552 pp.

Anderson, N. A., 1982. The genetics and pathology of Rhizoctonia solani. Ann. Rev. Phytopathol., 20: 329-344

Antralina, M., Istina, I.N., Yuwariah, Y. and Simarmata, T. 2015. Effect of different weed control methods to yield of lowland rice in The Sobari. Procedia Food Sci., 3: 323-329.

Bernardes-De-Assis, J., Storari, M., Zala, M., Wang, W. X., Jiang, D. H., Li, S. D. 2009. Genetic structure of population of the rice-infecting pathogen Rhizoctonia solani AG1-IA from China. Phytopathol., 99: 1090-1099.

Binder, M., Hibbett, D., Larsson, K., Larsson, E., Langer, E. and Langer, G. 2005. The phylogenetic distribution of resupinate forms across the major clades of mushroom-forming fungi (Homobasidiomycetes). Syst. Biodiver., $3(2): 1-45$

Biswas, G. and Mohanty, A.K. 1995. New host record for Drechslera oryzae. Indian J. Mycol. Plant Pathol., 25: 336.

Brar, J.S. and Thind, B.S. 1994. Anew weed host of Xanthomonas oryzae pv. oryzae, the causal agent of bacterial leaf blight of rice. Anna. Plant Prot. Sci., 2(1): 7980.

Carter, L. L. A., Leslie, F. J. and Webster, R. K. 2008. Population structure of Fusarium fujikuroi from California rice and water grass. Phytopathol., 98: 99298.

Deng, Q., Yong, M., Li, D., Lai, C., Chen, H., Fan, J. and Hu, D. 2015. Survey and examination of the potential alternative hosts of Villosiclava virens, the pathogen of rice false smut, in China. J. Integ. Agric., 14(7): 1332-1337.

Gonzaliz-Vera, A. D., Bernardes-De-Assis, J., Zala, M., McDonald, B. A., CorreaVictoria, F., Graterol-Matute, E. J. 2010. Divergence between sympatric rice and maize-infecting populations of Rhizoctonia solani AG1-IA from Latin America. Phytopathol., 100:172-182.

Hartman, G.L., Manandhar, J.B. and Sinclair, J.B. 1986. Incidence of Colletotrichum spp. On soybeans and weeds in Illinois and pathogenicity of Colletotrichum truncatum, Plant Dis., 70: 780-782.

Hosoya, K. and Sugiyama, S. 2017. Weed communities and their negative impact on rice yield in no-input paddy fields in the northern part of Japan. Biol. Agric. Hort., 33(4): 215-224.

IRRI., 1996. Standard Evaluation System for rice. INGER Genetic Resource Centre, 4th Edn. July, 1996.

Kannaiyan. S. and N.N. Prasad (1980). Dicot weed hosts of Rhizoctonia solani Kuhn. 
Agri. Res. J. Kerala, 18:125-127.

Kozaka, T., 1961. Ecological studies on sheath blight of rice plant caused by Pellicularia sasakii (Shirai) S. Ito, and its chemical control. Chugoku Agric. Res., 20:1-133.

Kozaka, T., 1965. Ecology of Pellicularia sheath blight of rice plant and its chemical control. Ann. Phytopathol. Soc. Japan, 31: 171-185.

Kumar, S., Lal, M., Garkoti, A. and Tripathi, H.S. 2013. Standardization of inoculation techniques, plant age and host range of Rhizoctonia solani, the incident of web blight of urdbean. Plant Dis. Res., 28 (1):45-48.

Ou, S. H., 1972. Rice diseases. First edition. The common wealth Mycological Institute, Kew Survey, England. 368pp.

Rai, M. 2006., Rice- the cereal that feeds billions. Indian Farm., 56 (7): 4-9.

Rajan, C.P.D., 1987. Estimation of yield loss due to sheath blight of rice. Indian Phytopathol., 40: 174-177.

Roy, A.K., 1973. Natural occurrence of Corticum sasakii on some weeds. Curr. Sci., 42: 842-843.

Sharma, B.D. and Murkharji, S.K. 1978. Natural occurrence of Corticium sasakii on four hosts. Sci. Cult., 44:53.

Shetty, S.A. and Shetty, H.S. 1985. An alternative host for Ustilaginodea virens (Cooke) Takahashi, IRRI Newsletter, 10: 11 .

Singh, R., Sunder, S. and Dodan, D.S. 2012. Status and weed hosts of Rhizoctonia solani Kuhn, the incitant of sheath blight of rice in Haryana. Plant Dis. Res., 27(2):225-228.

Srinivas, P., Ramesh Babu, S. and Ratan, V. 2014. Role of sclerotia, plant debris and different hosts on survival of rice sheath blight pathogen, Rhizoctonia solani. Int. J. Appl. Biol. Pharma. Tech., 5(2): 2933.

Sureshkumar, R., Reddy, Y.A. and Ravichandran, S. 2016. Effect of weeds and their management in transplanted rice- A review. Inter. J. Res. Appl. Nat. Soc. Sci., 4(11): 165-180.

Taheri, P. and Hofte, M. 2007. Riboflavininduced resistance against sheath blight of rice functions through the potentiation of lignin formation \& Jasmonic acid signalling pathway. Commun. Agric. Appl. Biol. Sci., 72: 309-313.

Tsai, W.H. 1974. Assessment of yield losses due to rice sheath blight at different inoculation stages. Taiwan Agric. Res., 23: 188-194.

Wu, W., Nie, L., Shah, F., Liao, Y., Cui, K., Jiang, D., Xie, J., Chen, Y. and Huang, J. 2013. Influence of canopy structure on sheath blight epidemics in rice. Plant Pathol., 63(1): 98-108.

Wu, W., Shah, F., Shah, F. and Huang, J. 2015. Rice sheath blight evaluation as affected by fertilization rate and planting density. Aust. Plant Pathol., 44(2): 183-189.

\section{How to cite this article:}

Mohd Ali, Ramji Singh, Mehi Lal, Sorabh Chaudhary, Santosh Kumar and Shameem Ahmad. 2019. Determination the Role of Weeds Hosts in Spreading of Sheath Blight from Weeds to Rice Crop in Western Plain Zone of Uttar Pradesh, India. Int.J.Curr.Microbiol.App.Sci. 8(09): 972-982. doi: https://doi.org/10.20546/ijcmas.2019.809.115 